\title{
1 Using honey to heal diabetic foot ulcers
}

3 P. C. Molan B.Sc. Ph.D.

4 Professor of Biological Sciences, and Director of the Honey Research Unit, Department of

5 Biological Sciences, University of Waikato, Hamilton, New Zealand

\section{J. A. Betts NP, RN, AdDipN, MN(Hons)}

8 Nurse Practitioner ${ }^{\mathrm{TM}}$ wound care, Waikato District Health Board, Hamilton, New Zealand

10 Corresponding author:

11 Professor P. C. Molan

Department of Biological Sciences

University of Waikato

Private Bag 3105

Hamilton 3240

New Zealand

17 Home address:

\section{Acacia Crescent}

Hamilton 3206

New Zealand 


\section{Abstract}

27 Diabetic ulcers seem to be arrested in the inflammatory/proliferative stage of the healing

28 process, allowing infection and inflammation to preclude healing. Antibiotic-resistant bacteria

29 have become a major cause of infections, including diabetic foot infections. It is proposed here

30 that the modern developments of an ancient and traditional treatment for wounds, dressing

31 them with honey, provide the solution to the problem of getting diabetic ulcers to move on from

32 the arrested state of healing. Honeys selected to have a high level of antibacterial activity have

33 been shown to be very effective against antibiotic-resistant strains of bacteria in laboratory and

34 clinical studies. The potent anti-inflammatory action of honey is also likely to play an important

35 part in overcoming the impediment to healing that inflammation causes in diabetic ulcers, as is

36 the antioxidant activity of honey. The action of honey in promotion of tissue regeneration

37 through stimulation of angiogenesis and the growth of fibroblasts and epithelial cells, and its

38 insulin-mimetic effect, would also be of benefit in stimulating the healing of diabetic ulcers. The

39 availability of honey-impregnated dressings which conveniently hold honey in place on ulcers

40 has provided a means of rapidly debriding ulcers and removing the bacterial burden so that

41 good healing rates can be achieved with neuropathic ulcers. With ischemic ulcers, where

42 healing cannot occur because of lack of tissue viability, these honey dressings keep the ulcers

43 clean and prevent infection occurring.

\section{Introduction}

46 In the recently published paper "What is the future of diabetic wound care?"- the authors give an

47 excellent review of the reasons why diabetic wounds do not heal, and provide data on the

48 magnitude of the problem this causes. They point out that many of the nearly 2 million cases of

49 chronic diabetic foot ulcers in the USA ultimately result in amputation, with a 2-year survival rate

50 after these amputations of only $50 \%-60 \%$. They then go on to discuss at length the need for

51 new smart matrix therapies, and how the development of these will require a multidisciplinary 
52 translational research approach. Considering that the research on tissue-engineered skin

53 substitutes was started approximately 20 years ago yet this form of therapy has barely started to

54 get into regular clinical practice, there is obviously an immediate need for an effective therapy

55 for chronic diabetic ulcers. It is proposed here that the modern developments of an ancient and

56 traditional treatment for wounds, dressing them with honey, have allowed this immediate need

57 to be met.

59 Historical usage of honey

60 The ancient Egyptians, Assyrians, Chinese, Greeks and Romans all used honey, in combination

61 with herbs and on its own, to treat wounds. ${ }^{2}$ Aristotle (c.350 BC) wrote of honey being a salve

62 for wounds and sore eyes,,$\underline{3}$ and Dioscorides (c.50 AD) wrote of honey being "good for sunburn

63 and spots on the face" and "for all rotten and hollow ulcers". 4 The usage of honey has continued

64 into present-day folk-medicine; for example, it is a traditional therapy for infected leg ulcers in

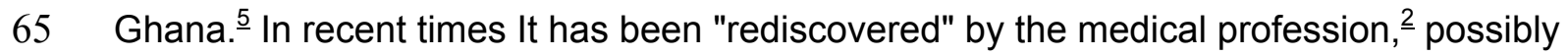

66 because the "antibiotic era" is coming to an end as increasing numbers of bacterial strains

67 develop resistance to antibiotics. $\underline{6}$

\section{Modern clinical evidence for the effectiveness of honey}

70 The clinical evidence in support of the effectiveness of honey in wound care has been

71 comprehensively reviewed. ${ }^{7}$ This review summarises the findings of 17 randomised controlled

72 trials involving a total of 1965 participants, and 5 clinical trials of other forms involving 97

73 participants treated with honey. All of these found that honey was more effective than the

74 conventional wound care practices used as controls, other than one trial on burns where, only in

75 respect of control of infection, was early surgical tangential incision found to be more effective

76 than dressing the wounds with honey. The review also summarises the findings 16 trials on a

77 total of 533 wounds on experimental animals, where again honey was found to be more 
78 effective than the controls in assisting wound healing. There is also summarised a large amount

79 of evidence in the form of case studies that have been reported. Ten publications have reported

80 on multiple cases, totalling 276 cases. There are also 35 reports of single cases. ${ }^{7}$ This evidence

81 is far greater than that for modern wound dressings. It is possibly the myriad of advertisements

82 for modern wound dressings, and little for honey, that gives clinicians the impression that there

83 is much evidence is behind the modern dressings and none behind honey, when in fact only

84 poor-quality trials exist to support the modern wound dressings that are in use. $\stackrel{8}{\text { Perhaps the }}$

85 most heavily advertised wound dressings are the nanocrystalline silver dressings, but if the

86 PubMed database is searched for evidence to support their use it can be seen that there is in

87 fact very little good clinical evidence that has been published other than two recent trials. A

88 conclusion reached in a recent systematic review of publications on the use of advanced

89 dressings in the treatment of pressure ulcers has found that their generalised use in the

90 treatment of pressure ulcers is not supported by good research evidence. ${ }^{9}$ There is now a

91 general movement towards evidence-based medicine. In this, decisions should be made on the

92 basis of the available evidence. If randomised controlled trials of the highest quality have not

93 been conducted, then it is necessary to consider evidence of a lower quality. Advertising, other

94 than that which presents good clinical evidence, should not be allowed to influence decisions.

96 Usage of honey on diabetic ulcers

97 Although there is a vast amount of literature reporting the effectiveness of honey in healing

98 wounds, there has been little published specifically on the treatment of diabetic foot ulcers with

99 honey. Diabetic ulcers are chronic wounds, and there is a large amount of evidence for the

100 effectiveness of honey on chronic wounds. Seven clinical trials, with a total of 255 participants,

101 have been conducted using honey on chronic wounds, with positive results for honey being

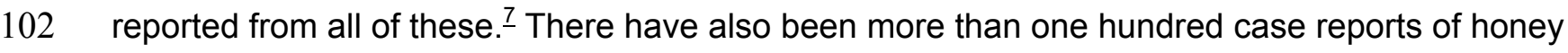

103 being more effective than conventional treatment for chronic wounds. ${ }^{7}$ Diabetic ulcers were 
104 included in one report in the list of recalcitrant wounds and ulcers of varied aetiology that

105 "showed remarkable improvement" when treated with honey. These were all wounds showing

106 no signs of healing, or were increasing in size, after 1-24 months of conventional treatment. $\frac{10}{1 n}$

107 another report, on using honey to treat septic wounds, chronic ulcers, burns, and pyogenic

108 abscesses, 6 patients were diabetic, 5 having a septic foot and 1 with an abscess in the thigh. 11

109 Healing time was 7, 10, 14 and 30 days for four the foot wounds, 15 days for the abscess, and

110 no improvement after 56 days for a foot wound on one patient who was ill. Another report, on a

111 single case, was on treatment of diabetic foot ulcers, $8 \times 5 \mathrm{~cm}$ and $3 \times 3 \mathrm{~cm}$, on a 79 year old

112 patient. $\frac{12}{}$ The ulcers remained unhealed after 14 months treatment with an orthotic device,

113 antibiotics, topical therapies by a wound care expert and four lots of surgery. MRSA, VRE and

114 Pseudomonas were present in wound tissue. After commencing treatment with honey, the

115 ulcers were granulating within 2 weeks, and healed within 6 and 12 months. Two years later the

116 ulcers had not recurred. Although honey gave healing of these ulcers where nothing else had

117 worked, the healing was still fairly slow. This possibly because "supermarket honey" was used,

118 which is honey that is usually processed in a way that destroys all of the antibacterial activity

119 and possibly removes other beneficial components. In the other two reports unprocessed honey

120 was used, which would have had its therapeutic properties undamaged.

\section{Antibacterial activity of honey}

123 There have been many publications on laboratory studies showing that honey has very broad

124 spectrum antibacterial activity. $\frac{13}{3}$ But most of these do not take into account that honeys can

125 vary as much as 100 -fold in the potency of their antibacterial activity. $\frac{14}{}$ Only in the more recent

126 publications was the testing of the sensitivity of various species of bacteria to honey done using

127 honeys selected to have antibacterial potency near the median level found in surveys of large

128 numbers of samples. Laboratory studies with Manuka (Leptospermum scoparium) honey with

129 antibacterial potency near the median level for active Manuka honey have shown the MIC 
130 (minimum inhibitory concentration, i.e. the concentration down to which honey could be diluted 131 by wound exudate and still prevent bacterial growth) to be 2-3\% for Staphylococcus aureus, $\frac{15}{-3}$

$1323.3-4 \%$ for coagulase-negative staphylococci, $\frac{16}{6.5-9 \%}$ for Pseudomonads,,$\stackrel{17,18}{2.7-3 \%}$ for

133 MRSA, $\underline{19}$ and $3.8-5 \%$ for VRE. $\stackrel{19}{ }$ The antibacterial potency of the honeys used in the various

134 honey wound-care products now on sale manufactured from Leptospermum honey (Manuka

135 honey and Jellybush honey) is higher than that of the honey used these studies, but there are

136 other wound-care products manufactured from honeys not selected to have high levels of

137 antibacterial activity. $\underline{\underline{20}}$

139 Another consideration is the type of antibacterial activity in honey. Most honeys owe their 140 antibacterial activity (beyond that just due to the osmotic effect of the high sugar content) to the

141 production of hydrogen peroxide by an enzyme that the bees secrete into the nectar harvested

142 to make honey. $\frac{13}{-}$ But some Manuka and Jellybush honeys have also a high activity due to a

143 phytochemical antibacterial component which comes from the nectar of some varieties of

144 Leptospermum. Although in laboratory testing the MIC values recorded are near the same for

145 mid-range honeys of either type, this would not be the case on a wound. There is in wound fluid,

146 and more so the cells in wound tissues, the enzyme catalase which rapidly breaks down

147 hydrogen peroxide, so honeys other than the Leptospermum ones would have their antibacterial

148 activity greatly decreased. Also to be noted is that all types of honey vary from batch to batch in

149 their antibacterial potency. Much of the Manuka and Jellybush honey on sale as a food item has

150 little or none of the phytochemical antibacterial component, but the Leptospermum honey on

151 sale as medical products has standardised high levels of it.

\section{Why honey works well on diabetic ulcers}

154 A wound will not heal if it is infected. Toxins produced by bacteria can stop the growth of cells 155 which repair the wound. Bacterial proteases can digest away the matrix necessary for tissue 
156 replacement, and digest cell growth factors. Also, components of the outer coat of the bacteria 157 can stimulate a strong inflammatory response and the resultant inflammation then prevents 158 healing and if severe can activate proteases to cause ulceration. Foot infections are a serious 159 complication of diabetes and a leading cause of diabetes-related hospitalizations. Amputation 160 may be needed when infections fail to respond to therapy. The development by bacteria of 161 resistance to antibiotics has become a major global problem. Methicillin-resistant

162 Staphylococcus aureus (MRSA) has become a major cause of infections, including diabetic foot 163 infections. Initially it was associated only with hospital-acquired infections, but now is also a 164 problem in the community. The effectiveness of honey in clinical usage in clearing infection with 165 MRSA $\frac{12,21-24}{2}$ and $\mathrm{VRE}^{12}$ has been reported. As well the antibacterial action, there have been 166 many reports of honey having a rapid debriding effect, which painlessly lifts off slough and 167 necrotic tissue (which are reservoirs of bacteria on the wound), making surgical debridement 168 unnecessary. $\underline{25}$

170 Diabetic ulcers, instead of going through the normal four overlapping stages of healing, 171 hemostasis, inflammation, proliferation and remodelling, seem instead to be arrested in the 172 inflammatory/proliferative process. ${ }^{1}$ This allows for infection and inflammation, which preclude 173 healing. The potent anti-inflammatory action of honey is likely to play an important part in 174 overcoming this impediment to healing in diabetic ulcers. The anti-inflammatory action of honey 175 was noted by Dioscorides two millennia ago, who wrote of honey being good for sun-burn, ${ }^{4}$ and 176 since then a large amount of clinical and histological evidence for it has accumulated, which has

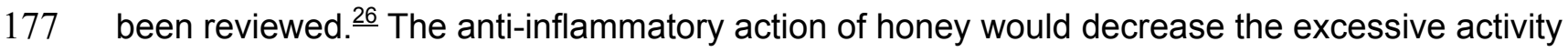
178 of collagenase and elastase, a feature of inflammatory conditions and which causes the 179 premature degradation of collagen and growth factors in chronic wounds. ${ }^{1} \mathrm{~A}$ characteristic of 180 the extracellular matrix in wounds in diabetes is the presence of advanced glycation and 181 lipoxidation end products which stimulate oxidative stress and inflammation in wounds. ${ }^{1}$ The 
182 excellent antioxidant properties of honey would be beneficial in counteracting these effects. It is 183 of note in this respect that another advantage of using active Manuka honey instead of other

184 types of honey is that it does not generate hydroxide free radicals like honey with antibacterial 185 activity due to hydrogen peroxide does, and rapidly quenches any formed by the Fenton 186 reaction. $\underline{27}$

188 The good results achieved on diabetic ulcers with honey may also be due to honey directly 189 moving the wounds on from the arrested state of healing. It has been observed that honey 190 promotes tissue regeneration through stimulation of angiogenesis and the growth of fibroblasts 191 and epithelial cells. $\underline{25}$

193 The slow healing rates seen in diabetic wounds could also be due to lack of insulin or lack of 194 responsiveness to insulin. As well as regulating the uptake of glucose into cells, insulin

195 functions as a growth hormone, and it has been shown that intravenous infusion or topical 196 application of insulin improves the rate of wound healing. $\frac{28-30}{}$ Honey has an insulin-mimetic

197 effect: when administered by inhalation as an aerosol, honey significantly reduced random 198 blood glucose (measured $30 \mathrm{~min}$ after inhalation), significantly reduced the fasting blood 199 glucose level (3 hours after inhalation), and the intensity of hyperglycemia was significantly 200 lowered in the glucose tolerance test after honey inhalation. $\underline{31}$

\section{Modern developments in the usage of honey}

204 In the "rediscovery" of the use of honey for wound care there has been a lack of awareness of 205 some ancient wisdom. Four millennia ago the Ancient Egyptians mixed cotton fibres and fat into 206 honey to create a dressing that would hold the honey on a wound. $\frac{32}{1}$ it is necessary to create a 207 retentive dressing of some sort, because at body temperature honey becomes very runny and 
208 flows off a wound. It takes very little dilution of honey with wound exudate to make the honey 209 very watery. The water content of honey is bound up on the sugar molecules in honey, so it 210 does not easily wet absorbent dressing materials. This means that if honey is applied to a 211 wound, whether directly or placed on an absorbent dressing, when the dressing is held on the

212 wound the honey tends to be squeezed out laterally. To get the honey to be retained well on the 213 wound it needs to be soaked into the absorbent dressing before it is applied. Various sorts of

214 such honey-impregnated dressings are now available as sterile medical products. $\underline{20}$

216 In our initial work on clinical use of honey dressings we used a Gamgee type of dressing

217 impregnated with honey. We found that with heavily exuding wounds it was necessary to 218 change the dressing up to three times a day, as the exudate flushed the honey from the side of

219 the dressing facing the wound to the outside of the dressing pad or even right through it and into 220 the secondary dressing. This left no honey in contact with the wound bed, with the consequence 221 that healing was slow, adhesion of the dressings occurred, and maceration of skin around the 222 wound occurred. This situation was greatly improved when we changed to using a honey223 impregnated alginate fibre dressing pad instead, as the alginate fibres transform to a gel when 224 wound exudate is absorbed into them. (This also has the advantage of the hemostatic 225 properties of alginate.) Thus the honey is held in a soft gel in contact with the wound bed rather 226 than being flushed into the secondary dressing, so less frequent changes of dressings are 227 needed. By keeping sufficient honey in contact with the wound bed, by changing the dressings 228 frequently enough to cope with the level of exudate, the level of exudate will decrease because 229 of the anti-inflammatory activity of honey. Less frequent dressing changes will be needed then. 230 However, if dressings are not changed frequently enough and the honey is washed away, then 231 the anti-inflammatory activity will also be washed away and the wound will remain highly 232 exudative. Another form of dressing has been developed in which the honey is combined with 233 sodium alginate to gel it, giving a rubbery material which swells as it absorbs exudate. $\frac{33}{-}$ This 
234 has a massive capacity to absorb exudate without the honey being flushed from the wound bed.

235 Commercialisation of this dressing is proceeding, and it is expected to be available for clinical

236 evaluation soon.

238 By updating the ancient wisdom of using a honey selected as the best for medical use (we use

239 active Manuka honey with a high level of the non-peroxide antibacterial activity that is unique to

240 Leptospermum honey, processed with minimal heating to avoid damaging its therapeutic

241 properties) and creating a dressing material to hold the honey in place on the wound (we use

242 alginate dressings pads impregnated with this honey) we have been able to get very good

243 results with diabetic ulcers. We use honey dressings on neuropathic foot ulcers where despite

244 pressure off-loading healing is inhibited by the presence of Pseudomonas and Staphylococcus

245 aureus. We use these dressings to debride the ulcers and reduce the bacterial burden. Good

246 healing rates are then achieved. With ischaemic foot ulcers, where healing cannot occur

247 because of lack of tissue viability, we use honey dressings to prevent infection of these ulcers

248 whilst waiting for re-vascularisation to be carried out. We find that if there is enough blood flow

249 then diabetic ulcers which are due to a combination of ischemia and neuropathy will heal with

250 off-loading of pressure. We find that honey is particularly useful for diabetic ulcers that are

251 otherwise difficult to debride particularly where bacterial loading may be a confounding factor in

252 the creation of slough.

\section{References}

255 1. Sweitzer SM, Fann SA, Borg TK, Baynes JW, Yost MJ. What is the future of diabetic wound 256 care? Diabetes Educ 2006;32(2):197-210.

257 2. Zumla A, Lulat A. Honey - a remedy rediscovered. J R Soc Med 1989;82(7):384-385. 
258 3. Aristotle. Historia Animalium. Oxford, U.K.: Oxford University; 1910. Volume IV

259 4. Gunther RT. The Greek Herbal of Dioscorides. New York: Hafner; 1934 (Reprinted 1959).

260 5. Ankra-Badu GA. Sickle cell leg ulcers in Ghana. East Afr Med J 1992;69(7):366-369.

261 6. Greenwood D. Sixty years on: antimicrobial drug resistance comes of age. Lancet 262 1995;346(Supplement 1):s1.

263 7. Molan PC. The evidence supporting the use of honey as a wound dressing. Int J Low 264 Extrem Wounds 2006;5(1):40-54.

265 8. Vermeulen H, Ubbink DT, Goossens A, de Vos R, Legemate DA. Systematic review of 266 dressings and topical agents for surgical wounds healing by secondary intention. Br J Surg $267 \quad 2005 ; 92(6): 665-72$.

268 9. Bouza C, Saz Z, Muñoz A, Amate JM. Efficacy of advanced dressings in the treatment of 269 pressure ulcers: a systematic review. J Wound Care 2005;14(5):193-9.

270 10. Efem SEE. Clinical observations on the wound healing properties of honey. Br J Surg $271 \quad 1988 ; 75: 679-681$.

272 11. Farouk A, Hassan T, Kashif H, Khalid SA, Mutawali I, Wadi M. Studies on Sudanese bee 273 honey: laboratory and clinical evaluation. Int J Crude Drug Res 1988;26(3):161-168.

274 12. Eddy JJ, Gideonsen MD. Topical honey for diabetic foot ulcers. J Fam Pract $275 \quad 2005 ; 54(6): 533-5$.

276 13. Molan PC. The antibacterial activity of honey. 1. The nature of the antibacterial activity. Bee $277 \quad$ World 1992;73(1):5-28. 
278 14. Molan PC. The antibacterial activity of honey. 2. Variation in the potency of the antibacterial 279 activity. Bee World 1992;73(2):59-76.

280 15. Cooper RA, Molan PC, Harding KG. Antibacterial activity of honey against strains of 281 Staphylococcus aureus from infected wounds. J R Soc Med 1999;92(6):283-285.

282 16. French VM, Cooper RA, Molan PC. The antibacterial activity of honey against coagulase283 negative staphylococci. J Antimicrob Chemother 2005;56(1):228-31.

284 17. Cooper RA, Halas E, Molan PC. The efficacy of honey in inhibiting strains of Pseudomonas aeruginosa from infected burns. J Burn Care Rehabil 2002;23(6):366-370.

18. Cooper RA, Molan PC. The use of honey as an antiseptic in managing Pseudomonas infection. J Wound Care 1999;8(4):161-164.

19. Cooper RA, Molan PC, Harding KG. The sensitivity to honey of Gram-positive cocci of clinical significance isolated from wounds. J Appl Microbiol 2002;93:857-863.

20. Molan PC, Betts JA. Clinical usage of honey as a wound dressing: an update. J Wound $291 \quad$ Care 2004;13(9):353-6.

292 21. Dunford C, Cooper R, Molan PC, White R. The use of honey in wound management. Nurs 293 Standard 2000;15(11):63-68.

294 22. Natarajan S, Williamson D, Grey J, Harding KG, Cooper RA. Healing of an MRSA295 colonized, hydroxyurea-induced leg ulcer with honey. J Dermatolog Treat 2001;12:33-36.

296 23. Dunford CE. Treatment of a wound infection in a patient with mantle cell lymphoma. Br J $297 \quad$ Nurs 2001;10(16):1058-1065. 
298 24. Simon A, Sofka K, Wiszniewsky G, Blaser G, Bode U, Fleischhack G. Wound care with 299 antibacterial honey (Medihoney) in pediatric hematology-oncology. Support Care Cancer $300 \quad 2006 ; 14(1): 91-7$.

301 25. Molan PC. Potential of honey in the treatment of wounds and burns. Am J Clin Dermatol $302 \quad 2001 ; 2(1): 13-19$.

303 26. Molan PC. Re-introducing honey in the management of wounds and ulcers - theory and 304 practice. Ostomy/Wound Manage 2002;48(11):28-40.

305 27. Henriques A, Jackson S, Cooper R, Burton N. Free radical production and quenching in 306 honeys with wound healing potential. J Antimicrob Chemother 2006;58(4):773-777.

307 28. Pierre EJ, Barrow RE, Hawkins HK, et al. Effects of insulin on wound healing. J Trauma $308 \quad$ Injury Infect Crit Care 1998;44(2):342-345.

29. Belfield WO, Golinsky S, Compton MD. The use of insulin in open wound healing. Vet Med $310 \quad$ Small Anim Clin 1970;65(5):455-460.

311 30. Lopez JE, Mena B. Local insulin for diabetic gangrene. Lancet 1968;i:1199.

312 31. Al-Waili N. Intrapulmonary administration of natural honey solution, hyperosmolar dextrose 313 or hypoosmolar distill water to normal individuals and to patients with type-2 diabetes 314 mellitus or hypertension: their effects on blood glucose level, plasma insulin and C-peptide, 315 blood pressure and peaked expiratory flow rate. Eur J Med Res 2003;8(7):295-303.

316 32. Majno G. The Healing Hand. Man and Wound in the Ancient World. Cambridge, 317 Massachusetts: Harvard University Press; 1975. 
318 33. Molan PC, inventor Waikatolink Limited, assignee. Honey based wound dressing. United 319 States patent US 6,956,144 B2. 2005 Oct.18, 2005. 